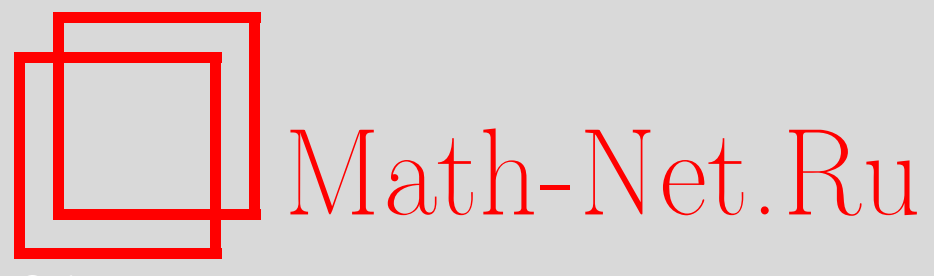

А. И. Штерн, Автоматическая непрерывность псевдохарактеров на полупростых группах Ли, Матем. заметки, 2006, том 80, выпуск 3, 456-464

DOI: https://doi.org/10.4213/mzm2832

Использование Общероссийского математического портала Math-Net.Ru подразумевает, что вы прочитали и согласны с пользовательским соглашением http://www . mathnet.ru/rus/agreement

Параметры загрузки:

IP: 54.84 .234 .179

26 апреля 2023 г., $16: 45: 42$

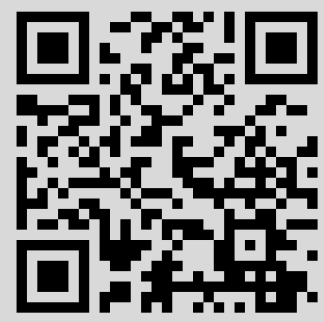




\section{АВТОМАТИЧЕСКАЯ НЕПРЕРЫВНОСТЬ ПСЕВДОХАРАКТЕРОВ НА ПОЛУПРОСТЫХ ГРУППАХ ЛИ}

\section{А. И. Штерн}

Показано, что любой псевдохарактер на полупростой группе Ли непрерывен. Библиография: 42 названия.

1. Введение. Постановка задачи. В то время как вопросы непрерывности представлений групп многократно изучались с различных точек зрения (как в предположении измеримости или борелевости этих представлений, если речь шла о представлениях локально компактных или метрических групп (см., например, [1]-[10]), так и без этого предположения, в том числе для более общих классов топологических групп [11]-[16]), аналогичный вопрос для квазипредставлений, т.е. таких отображений $\pi$ данной группы $G$ в группу обратимых операторов в некотором банаховом пространстве $E$, что величина

$$
\sup _{g_{1}, g_{2} \in G}\left\|\pi\left(g_{1} g_{2}\right)-\pi\left(g_{1}\right) \pi\left(g_{2}\right)\right\|_{E}
$$

мала, практически не исследован. Сложность этого вопроса связана с тем, что он не индивидуален. Вопрос относится, собственно, не к объекту, а к классу и ставится следующим образом: есть ли непрерывное отображение среди малых возмущений данного квазипредставления $\pi$ ?

Простейшим классом групп, в котором этот вопрос заслуживает изучения, является класс групп Ли. Для однопараметрических групп ответ на вопрос тривиальным образом отрицателен (достаточно упомянуть факт существования разрывных характеров, см., например, [17]), и поэтому он отрицателен и для разрешимых групп Ли, поскольку любая разрешимая группа Ли имеет однопараметрическую факторгруппу. Поэтому следует изучить вопрос о непрерывности для полупростых групп Ли. Поскольку каждая полупростая группа Ли является факторгруппой прямого произведения простых групп Ли, мы будем рассматривать условия автоматической непрерывности для простых групп Ли.

В этой статье мы рассмотрим простейший случай “логарифмируемых" одномерных квазипредставлений, т.е. экспонент от вещественных квазихарактеров на группе. Напомним некоторые определения, необходимые при формулировании основного результата. Вещественная функция $f$ на группе $G$ называется (вещественным)

Работа выполнена при частичной поддержке Российского фонда фундаментальных исследований, грант № 02-01-00574, и программы "Ведущие научные школы”, грант № НШ-619.2003.1.

(C) А. И. Штерн, 2006 
квазихарактером на этой группе [18], [19], если числовое множество

$$
\{f(g h)-f(g)-f(h) \mid g, h \in G\}
$$

ограничено. Кроме того, квазихарактер $f$ называется псевдохарактером на $G$, если

$$
f\left(g^{n}\right)=n f(g) \quad \text { для любых } \quad g \in G \quad \text { и } \quad n \in \mathbb{Z} .
$$

Понятие псевдохарактера было введено в 1983 г. [20]. Это понятие (применявшееся позже в ряде работ и под именем однородного квазиморфизма [21]) оказалось весьма продуктивным в теории ограниченных когомологий [22]-[28], в теории групп диффеоморфизмов [29], в симплектической геометрии [30]-[32], в комбинаторной теории групп [25] и в теории представлений групп [33], [34] и заслужило популярность, достаточную для отдельной пояснительной публикации в Notices AMS [35].

Напомним, что для любого квазихарактера $f$ на группе $G$ формула

$$
\varphi(g)=\lim _{n \rightarrow \infty} n^{-1} f\left(g^{n}\right), \quad g \in G,
$$

корректно определяет псевдохарактер $\varphi$ на $G$, который называется nсевдохарактером, связанным с квазихарактером $f$. Этот псевдохарактер $\varphi$ непрерывен, если $G-$ топологическая группа и квазихарактер $f$ непрерывен [18], [24]-[28], [36].

В отличие от общего случая квазипредставлений, любой квазихарактер определяет класс эквивалентности (класс отображений, получаемых ограниченным возмущением данного квазихарактера), допускающий выделенный элемент, а именно, псевдохарактер, связанный с данным квазихарактером (3). Таким образом, следующее утверждение можно рассматривать как первый шаг в изучении проблемы автоматической непрерывности для квазипредставлений (в форме, поставленной выше).

ТЕОРема 1. Любой псевдохарактер на простой группе Ли непрерывен.

Мы докажем это утверждение ниже, но сначала разъясним и уточним его.

Напомним, что простая группа Ли называется эрмитово симметрической, если центр ее универсальной накрывающей группы бесконечен (см. [37]). Семейство некомпактных простых эрмитово симметрических групп Ли состоит из связных простых групп Ли, присоединенная группа которых изоморфна присоединенной группе одной из следующих групп Ли (см. [37]):

(i) $\mathrm{SU}(p, q), p, q \in \mathbb{N}$;

(ii) $\mathrm{SO}_{0}(2, q)$, где $q \in \mathbb{N}, q \neq 2$ (напомним, что группа $\mathrm{SO}_{0}(2,2)$ не является простой);

(iii) $\operatorname{Sp}(n, \mathbb{R}), n \in \mathbb{R}$;

(iv) $\mathrm{SO}^{*}(2 n), n>1$ (напомним, что группа $\mathrm{SO}^{*}(2)$ не является простой);

(v) вещественная форма комплексной простой группы Ли типа $\mathrm{E}_{6}$, удовлетворяющая условию $\operatorname{dim} \mathfrak{k}=46$, где $\mathfrak{k}-$ алгебра Ли максимальной компактной подгруппы присоединенной группы этой вещественной формы;

(vi) вещественная форма комплексной простой группы Ли типа $\mathrm{E}_{7}$, удовлетворяющая условию $\operatorname{dim} \mathfrak{k}=79$, где $\mathfrak{k}-$ алгебра Ли максимальной компактной подгруппы присоединенной группы этой вещественной формы. 
Напомним далее, что односвязные эрмитово симметрические простые группы Ли - единственные простые группы Ли, допускающие нетривиальные псевдохарактеры. А именно, псевдохарактер Гишарде-Вигнера на односвязной эрмитово симметрической простой группе Ли $G$ (центр которой, тем самым, бесконечен) определяется следующим образом. Пусть $K$ - аналитическая подгруппа группы $G$, отвечающая максимальной компактной подалгебре Ли алгебры Ли группы Ли $G$. Осуществим изоморфное отождествление центра $Z_{K}$ аналитической группы $K$ с аддитивной группой поля вещественных чисел $\mathbb{R}$ (например, с помощью натурального параметра на кривой). Рассмотрим разложение Ивасавы группы $G$. Пусть $A-$ абелева, а $N$ - нильпотентная группа в этом разложении, и пусть

$$
g=k(g) a n, \quad g \in \widetilde{G}, \quad k(g) \in \widetilde{K}, \quad a \in A, \quad n \in N,
$$

- соответствующее разложение элемента $g \in G$. Как хорошо известно, отображение

$$
\varpi: g \mapsto k(g), \quad g \in G,
$$

переводящее каждый элемент $g \in G$ в "компактную" компоненту $k(g) \in K$ его разло-

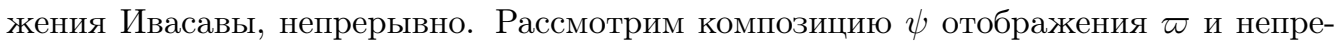
рывной проекции $\pi$, отображающей каждый элемент $k \in K$ в его центральную составляющую $z(k) \in Z_{K}$. Как доказано в [24]-[28], эта композиция

$$
\psi=\pi \circ \varpi
$$

определяет некоторый квазихарактер на $G$. Напомним также, что псевдохарактер $\varphi$, соответствующий квазихарактеру $\psi$ (см. (3)), мы называем псевдохарактером Гишарде-Вигнера [24]-[28]. Как отмечено выше, псевдохарактер Гишарде-Вигнера автоматически непрерывен на всей группе $G$, поскольку он связан с непрерывным квазихарактером $\psi$ формулой (3) (см. [24]-[28]).

Итак, сам предмет исследования, т.е. семейство псевдохарактеров на простой группе Ли, существенно зависит от того, является ли группа эрмитово симметрической. В связи с этим наблюдением теорема 1 может быть уточнена следующим образом.

ТеОРема 2. Пусть $G$ - простая группа Ли. Если $G$ не является эрмитово симметрической группой или если центр группы $G$ конечен, то любой псевдохарактер на $G$ тождественно равен нулю. Если $G$ - эрмитово симметрическая группа с бесконечным центром, то любой псевдохарактер на $G$ кратен псевдохарактеру Гишарде-Вигнера на $G$. В частности, любой псевдохарактер на простой группе Ли непрерывен.

Доказательство этой теоремы приведено в следующем разделе.

2. Доказательство теоремы 2. Доказательство теоремы будет дано после нескольких лемм и следствий.

ЛЕмма 1. Если $G$-аменабельная группа (см., например, [38]), то любой псевдохарактер на $G$ является обычным характером. В частности, ограничение любого псевдохарактера на коммутативную подгруппу является обычным характером этой подгруппъ. 
ДокАЗАТЕЛЬСтво. Утверждение леммы 1 - частный случай весьма общего факта, но мы напомним его доказательство для полноты изложения. Пусть $f-$ псевдохарактер на $G$. Из определения псевдохарактера следует, что функция

$$
t \rightarrow f(t s)-f(t), \quad t, s \in G,
$$

ограничена на $G$. Для любого правоинвариантного среднего $I$ на пространстве В $(G)$ ограниченных функций на $G$ положим

$$
\varphi_{I}(g)=I_{t}(f(t g)-f(t)), \quad g \in G .
$$

Тогда

$$
\begin{aligned}
\varphi_{I}(s w) & =I_{t}(f(t s w)-f(t))=I_{t}((f(t s w)-f(t s))+(f(t s)-f(t))) \\
& =\varphi_{I}(w)+\varphi_{I}(s), \quad s, t, w \in G,
\end{aligned}
$$

откуда следует, что $\varphi_{I}-($ аддитивный $)$ характер $G$. Это означает, в частности, что

$$
\varphi_{I}\left(g^{n}\right)=n \varphi_{I}(g) \quad \text { для всех } g \in G, \quad n \in \mathbb{N},
$$

так что либо $\varphi_{I}(s)=0$, либо множество $\left\{\varphi_{I}(s) \mid s \in G\right\}$ неограничено. С другой стороны, по условию,

$$
|f(t s)-f(t)-f(s)| \leqslant C \quad \text { для всех } t, s \in S \text {; }
$$

применяя среднее $I$ по $t$, видим, что

$$
\left|\varphi_{I}(s)-f(s)\right| \leqslant C \quad \text { для всех } \quad s \in S .
$$

Следовательно, $\varphi_{I}-f$ - ограниченный псевдохарактер, т.е. нулевая функция, именно потому, что

$$
\varphi_{I}(g)=n^{-1} \varphi_{I}\left(g^{n}\right) \quad \text { для всех } g \in G \text { и всех натуральных } n \text {. }
$$

Таким образом, $f$ - (обычный) вещественный характер группы $G$, что и утверждалось.

Повторяя рассуждение, легко видеть, что если $G$ - топологическая группа и если $G$ аменабельна как топологическая группа (см., например, [38]), то любой непрерывный псевдохарактер на $G$ является обычным характером. В частности, в этой ситуации ограничение любого непрерывного псевдохарактера на замкнутую коммутативную подгруппу является обычным непрерывным характером этой подгруппы. Отметим очевидное следствие этого наблюдения.

СлЕДСТВИЕ 1. Любой непрерывный псевдохарактер связной локально компактной группы тождественно равен нулю на любой компактной подгруппе этой груп$n b$.

Действительно, ограничение этого псевдохарактера на компактную подгруппу есть ее непрерывный (вещественный) характер; в частности, ограниченный. 
ЛЕмма 2. Если $G$ - полупростая связная группа Ли, не являющаяся эрмитово симметрической, то единственным псевдохарактером на $G$ является нулевой характер.

ДокАЗАТЕЛЬСтво. Из нашего предположения следует, что центр группы $G$ конечен. Пусть $f$ - псевдохарактер на $G$. Из леммы 1 следует, что ограничение $f$ на каждую однопараметрическую подгруппу в $G$ является ее гомоморфизмом. Кроме того, псевдохарактер $f$ инвариантен относительно внутренних автоморфизмов, так что его ограничения на торы (которые являются обычными гомоморфизмами по лемме 1) инвариантны относительно соответствующих групп Вейля.

Воспользуемся теперь разложением Ивасавы $G=K A N$, где группа $K$ компактна (поскольку центр группы $G$ конечен), а группы $A, N$ и $A N$ аменабельны (поскольку $A$ абелева, $N$ нильпотентна, а $A N$ разрешима). Из нашего предположения следует, что компактная группа $K$ полупроста. Если $f$ - псевдохарактер на $G$, то его ограничения на подгруппы $K$ и $A N$ являются соответственно псевдохарактерами $\varkappa_{K}$ и $\varkappa_{A N}$ на этих группах. По лемме 1 псевдохарактер $\varkappa_{A N}$ является обычным характером. Но любой характер группы $A N$ тривиален на нильпотентной части $N$, так что для $g=k a n$ разность

$$
f(g)-\varkappa_{K}(k)-\varkappa_{A N}(a)
$$

должна быть ограничена. С другой стороны, характер $\varkappa_{A N}$ должен быть инвариантен относительно группы Вейля, связанной с $A$. Эта группа Вейля содержит семейство отражений в элементах некоторого базиса, так что характер $\varkappa_{A N}$ должен принимать равные значения в точках, симметричных относительно соответствующих плоскостей. Гиперплоскостей, проходящих через эти орбиты относительно группы Вейля, не существует. Следовательно, характер $\varkappa_{A N}$ должен быть нулевым. Наконец, любой элемент полупростой компактной группы Ли может быть представлен в виде произведения ограниченного числа элементов, принадлежащих подгруппам, изоморфным группе $\mathrm{SU}(2)$ [39; 6.45 и 6.46]. Но любой элемент в группе $\mathrm{SU}(2)$ coпряжен своему обратному, и поэтому любой псевдохарактер на группе $\mathrm{SU}(2)$ равен нулю. Следовательно, любой псевдохарактер на полупростой компактной группе Ли ограничен и поэтому равен нулю. В частности, псевдохарактер $\varkappa_{K}$ нулевой. Таким образом, псевдохарактер $f$ ограничен, так что $f=0$, что и требовалось.

ЛЕмма 3. Если $G$ - эрмитово симметрическая полупростая связная группа Ли, то любой псевдохарактер на $G$ является одним из псевдохарактеров ГишардеВигнера на $G$. В частности, если центр группы $G$ конечен, то любой псевдохарактер на $G$ равен нулю.

Очевидно, не все непрерывные одномерные псевдопредставления такой группы тривиальны. Даже если $G$ - группа без центра (присоединенная), формула

$$
\varphi_{n}(g)=\exp \left(2 i n \pi \frac{f(\widetilde{g})}{f\left(z_{0}\right)}\right), \quad g \in G, \quad n \in \mathbb{Z},
$$

где $z_{0}$ - образующий элемент центра универсальной накрывающей $\widetilde{G}$ группы $G$ (см., например, [40]), $\widetilde{g}$ - любой прообраз элемента $g \in G$ в $\widetilde{G}$, а $f$ - характер ГишардеВигнера на $\widetilde{G}$, корректно определяет (нетривиальное при $n \neq 0$ ) одномерное псевдопредставление группы $G$ (ср. [33], [34]). 
Схема доказательства леммы 3 приведена в [41], и здесь мы следуем этой схеме. Докажем сначала следующее вспомогательное предложение.

Лемма 4. Пусть $G$ - односвязная эрмитово симметрическая простая группа Ли, $K$ - аналитическая подгруппа группы $G$, отвечающая максимальной компактной подалгебре Ли алгебры Ли группь Ли G. Рассмотрим разложение Ивасавъ

$$
g=k(g) a n, \quad g \in G, \quad k \in K,
$$

связанное с группой $K$. Рассмотрим композицию отображения $g \mapsto k(g), g \in G$, u непрерывной проекиии, отображающей каждый элемент $k \in K$ в его центральную составляющую $z(k) \in Z_{K}$. Существует класс сопряженных элементов, на котором эта композиция не принимает постоянного значения.

ДокАЗАтЕЛЬСтво. Если рассматриваемая композиция постоянна на классах сопряженных элементов, то компактная подгруппа $Q \subset K$ с тривиальной центральной компонентой в группе $K\left(\right.$ с $\left.z(k)=e_{K}\right)$ является нормальным делителем не только в $K$, но и в $G$, что невозможно.

ДоКАЗАТЕЛЬСТво ЛЕммы 3. Пусть $G$ - эрмитово симметрическая полупростая связная группа Ли и пусть $f$ - псевдохарактер на $G$. Пусть $Q \subset K$ - компактная подгруппа, образованная элементами с тривиальной центральной компонентой в группе $K\left(\right.$ с $\left.z(k)=e_{K}\right)$. По существу повторяя рассуждения, примененные в доказательстве леммы 2, мы видим, что ограничения псевдохарактера $f$ на $Q$ и $A N$ равны нулю. Таким образом, достаточно доказать, что ограничение псевдохарактера $f$ на центр $Z_{K}$ компактной группы $K$, которое является обычным характером центра по лемме 1, непрерывно. Действительно, если это ограничение непрерывно, то ограничения псевдохарактера $f$ на $K$ и на $A N$ совпадают с ограничениями некоторого псевдохарактера Гишарде-Вигнера на эти подгруппы. Тогда разность этих псевдохарактеров ограничена и является псевдохарактером, и поэтому равна нулю, так что рассматриваемый псевдохарактер действительно совпадает с некоторым псевдохарактером Гишарде-Вигнера.

Итак, нам осталось доказать, что обычный вещественный характер центра $Z_{K}$ группы $K$, получаемый при ограничении псевдохарактера $f$ на $Z_{K}$, непрерывен. Воспользуемся леммой 4. Согласно этой лемме существует класс сопряженных элементов, на котором композиция отображения $g \mapsto k(g), g \in G$, и непрерывной проекции, отображающей каждый элемент $k \in K$ в его центральную составляющую $z(k) \in Z_{K}$, не принимает постоянного значения. Следовательно, образ ограничения этого вещественно-аналитического отображения на класс сопряженных элементов, содержащий некоторый нетривиальный элемент вида $a n, a \in A, n \in N$, не является одноточечным множеством и, следовательно, содержит некоторый интервал $V$ в силу теоремы о сохранении области. Ограничение рассматриваемого характера на окрестность $V$ ограничено, поскольку произведение любого элемента $z$ окрестности $V$ на произведение некоторого элемента $q \in Q$ и некоторого элемента an $\in A N$ принадлежит выделенному классу сопряженных элементов, содержащем некоторый фиксированный элемент $z_{0} \in K_{T}$, так что на произведении zqan псевдохарактер $f$ принимает то же значение, что и на построенном элементе $z_{0}$ группы $Z_{K}$, причем

$$
\begin{aligned}
|f(z)| & \leqslant \mid f(z)-f(\text { zqan })|+| f(\text { zqan }) \mid \\
& =\mid f(z)-f(z)-f(q)-f(\text { an })|+| f\left(z_{0}\right)|+2 C \leqslant| f\left(z_{0}\right) \mid+2 C
\end{aligned}
$$


при $z \in V$, так что псевдохарактер $f$, а потому и характер, определяемый ограничением псевдохарактера $f$ на $Z_{K}$, ограничен на $V$. Однако, как хорошо известно, любой характер группы $\mathbb{R}$, ограниченный на некоторой окрестности, непрерывен ${ }^{1}$. Это завершает доказательство леммы 4.

\section{Доказательство теоремы 2 непосредственно следует из лемм 1-3.}

3. Заключительные замечания. Нетрудно убедиться, что теорема 2 допускает распространение (без существенных изменений) на случай любых одномерных квазипредставлений полупростых групп, откуда, в свою очередь, следует, что любое одномерное псевдопредставление полупростой группы является экспонентой от псевдохарактера этой группы. В сочетании с недавно полученным полным (положительным) решением проблемы Мильмана для полупростых компактных групп Ли (постановка этой проблемы приведена Кажданом в [42]), это позволяет доказать автоматическую непрерывность (в смысле, описанном во введении к статье) любых конечномерных квазипредставлений полупростых групп Ли, но доказательство этой теоремы непрерывности громоздко и не может быть приведено в рамках настоящей статьи.

Отметим интересную нерешенную задачу: верно ли утверждение теоремы 2 для любых связных групп Ли, действие полупростой факторгруппы которых на факторгруппе радикала по его коммутанту не имеет нетривиальных неподвижных точек?

\section{СПИСОК ЦИТИРОВАННОЙ ЛИТЕРАТУРЫ}

[1] S. Banach, Théorie des opérations linéaires, Monograf. Mat., 1, PWN, Warszawa, 1932.

[2] J.W. Baker, B. M. Lashkarizadeh, "Representations and positive definite functions on topological semigroups", Glasgow Math. J., 38:1 (1996), 99-111.

[3] R. Exel, M. Laca, "Continuous Fell bundles associated to measurable twisted actions", Proc. Amer. Math. Soc., 125:3 (1997), 795-799.

[4] S. A. Gaal, Linear Analysis and Representation Theory, Springer-Verlag, New YorkHeidelberg, 1973.

[5] C. C. Moore, "Group extensions and cohomology for locally compact groups. III", Trans. Amer. Math. Soc., 221:1 (1976), 1-33.

[6] R. T. Moore, Measurable, Continuous and Smooth Vectors for Semi-Groups and Group Representations, Mem. Amer. Math. Soc., 78, American Mathematical Society, Providence, RI, 1968.

[7] K.-H. Neeb, "On a theorem of S. Banach", J. Lie Theory, 7:2 (1997), 293-300.

[8] K.-H. Neeb, D. Pickrell, "Supplements to the papers entitled: "On a theorem of S. Banach" and "The separable representations of $\mathrm{U}(H)$ "”, J. Lie Theory, 10:1 (2000), 107-109.

[9] V. Pestov, "Review of [7]", Math. Review 98i:22003, 1998.

[10] Z. Sasvari, Positive Definite and Definitizable Functions, Mathematical Topics, 2, Akademie Verlag, Berlin, 1994.

\footnotetext{
${ }^{1}$ Профессор Р. С. Исмагилов любезно сообщил мне, что аналогичное утверждение было доказано им в одной из его неопубликованных ранних работ в более сильной форме, а именно, для непрерывности характера достаточно его ограниченности на множестве положительной меры. В настоящее время техника развита настолько, что равносильность этого условия условию ограниченности на окрестности очевидна. Действительно, если вещественный характер ограничен на множестве $A$ локально компактной группы $G$, то он ограничен и на $A A^{-1}$, а такое множество содержит непустое открытое подмножество по теореме о непрерывности свертки двух квадратично суммируемых функций на локально компактной группе.
} 
[11] A. I. Shtern, "Continuity of Banach representations in terms of point variations", Russ. J. Math. Phys., 9:2 (2002), 250-252.

[12] А. И. Штерн, "Критерии слабой и сильной непрерывности представлений топологических групп в банаховых пространствах", Матем. сб., 193:9 (2002), 139-156.

[13] A.I. Shtern, "Continuity criteria for finite-dimensional representations of compact connected groups", Adv. Stud. Contemp. Math. (Kyungshang), 6:2 (2003), 141-156.

[14] A. I. Shtern, "Continuity conditions for finite-dimensional representations of some compact totally disconnected groups", Adv. Stud. Contemp. Math. (Kyungshang), 8:1 (2004), 1322.

[15] А. И. Штерн, "Критерии непрерывности конечномерных представлений связных локально компактных групп", Матем. сб., 195:9 (2004), 145-159.

[16] А.И. Штерн, "Критерий непрерывности конечномерных представлений локально компактных групп", Матем. заметки, 75:6 (2004), 951-953.

[17] Э. Хьюитт, К.А. Росс, Абстрактный гармонический анализ, 1, Наука, М., 1975.

[18] А. И. Штерн, "Квазипредставления и псевдопредставления", Функцион. анализ и прилож., 25:2 (1991), 70-73.

[19] A. I. Shtern, "Quasi-symmetry. I", Russ. J. Math. Phys., 2:3 (1994), 353-382.

[20] А. И. Штерн, "Устойчивость представлений и псевдохарактеры", Ломоносовские чтения, МГУ, М., 1983.

[21] C. Bavard, "Longueur stable des commutateurs", Enseign. Math. (2), 37:2 (1991), 109150.

[22] M. Gromov, "Positive curvature, macroscopic dimension, spectral gaps and higher signatures", Functional Analysis on the Eve of the 21st Century, Vol. II (New Brunswick, NJ, 1993), Progr. Math., 32, Birkhäuser, Boston, MA, 1996, 1-213.

[23] J. F. Manning, "Geometry of pseudocharacters", Geom. Topol., 9 (2005), 1147-1185.

[24] A. I. Shtern, Remarks on pseudocharacters and the real continuous bounded cohomology of connected locally compact groups, Sfb 288 Preprint № 289, 1997.

[25] А. И. Штерн, "Структурные свойства и ограниченные вещественные непрерывные 2когомологии локально компактных групп", Функцион. анализ и прилож., 35:4 (2001), $67-80$.

[26] A. I. Shtern, "Bounded continuous real 2-cocycles on simply connected simple Lie groups and their applications", Russ. J. Math. Phys., 8:1 (2001), 122-133.

[27] A. I. Shtern, "Remarks on pseudocharacters and the real continuous bounded cohomology of connected locally compact groups", Ann. Global Anal. Geom., 20 (2001), 199-221.

[28] A. I. Shtern, "A criterion for the second real continuous bounded cohomology of a locally compact group to be finite-dimensional", Acta Appl. Math., 68:1-3 (2001), 105-121.

[29] J.-M. Gambaudo, É. Ghys, "Commutators and diffeomorphisms of surfaces", Ergodic Theory Dynam. Systems, 24:5 (2004), 1591-1617.

[30] P. Biran, M. Entov, L. Polterovich, "Calabi quasimorphisms for the symplectic ball", Commun. Contemp. Math., 6:5 (2004), 793-802.

[31] D. Kotschick, S. Morita, "Signatures of foliated surface bundles and the symplectomorphism groups of surfaces", Topology, 44:1 (2005), 131-149.

[32] D. McDuff, "A survey of the topological properties of symplectomorphism groups", Topology, Geometry and Quantum Field Theory, London Math. Soc. Lecture Note Ser., 308, Cambridge Univ. Press, Cambridge, 2004, 173-193.

[33] А. И. Штерн, “Деформация неприводимых унитарных представлений дискретной серии эрмитово симметрических простых групп Ли в классе чистых псевдопредставлений", Матем. заметки, 73:3 (2003), 478-480.

[34] А. И. Штерн, "Проективные представления и чистые псевдопредставления эрмитово симметрических простых групп Ли", Матем. заметки, 78:1 (2005), 140-146.

[35] D. Kotschick, "What is... a quasi-morphism?", Notices Amer. Math. Soc., 51:2 (2004), 208-209. 
А. И. ШТЕРН

[36] A.I. Shtern, "Triviality and continuity of pseudocharacters and pseudorepresentations", Russ. J. Math. Phys., 5:1 (1997), 135-138.

[37] С. Хелгасон, Дифференциальная геометрия и симметрические пространства, Мир, M., 1964.

[38] A. L. T. Paterson, Amenability, Math. Surveys Monographs, 29, Amer. Math. Soc., Providence, RI, 1988.

[39] K. H. Hofmann, S. A. Morris, The Structure of Compact Groups, de Gruyter Studies in Math., 25, de Gruyter, Berlin, 1998.

[40] M. A. Naimark, A. I. Štern [Shtern], Theory of Group Representations, Springer-Verlag, New York-Heidelberg-Berlin, 1982.

[41] A. I. Shtern, "Automatic continuity of pseudocharacters on Hermitian symmetric semisimple Lie groups and some applications", Adv. Stud. Contemp. Math. (Kyungshang), 12:1 (2006), 1-8.

[42] D. Kazhdan, "On ع-representations", Israel J. Math., 43:4 (1982), 315-323.

\section{А. И. Штерн}

